\title{
A falling-pressure method for measuring air permeability of asphalt in laboratory
}

\author{
Hailong $\mathrm{Li}^{\mathrm{a}, \mathrm{b}, *}$, Jiu Jimmy Jiao ${ }^{\mathrm{b}}$, Mario $\mathrm{Luk}^{\mathrm{c}}$ \\ ${ }^{a}$ Department of Mathematics, Anshan Normal University, Anshan 114005, China \\ ${ }^{\mathrm{b}}$ Department of Earth Sciences, University of Hong Kong, Hong Kong, China \\ ${ }^{\mathrm{c}}$ Airport Authority Hong Kong, 8 Chun Yue Road, Hong Kong International Airport, Lantau, Hong Kong, China
}

Received 27 August 2002; revised 19 August 2003; accepted 12 September 2003

\begin{abstract}
This paper presents a simple analytical solution for estimating air permeability using the test data obtained by a fallingpressure method in laboratory. The perimeter of the column-shaped sample is fixed in a steel cylinder with the upper sample surface open to the atmosphere. The lower surface of the sample and the cylinder form an air chamber. A water manometer is connected to the air chamber to measure the air pressure inside after the chamber is pressurized. The data of pressure versus time in the air chamber are recorded and analyzed. An approximate analytical solution is derived to describe the pressure-time relationship in the air chamber. The air permeability can be easily estimated using the approximate analytical solution based on the linear least-squares fitting to the recorded pressure-time test data. This method is used to estimate the falling-pressure test data of 15 asphalt samples. The agreement between the test data and the analytical prediction is satisfactory for all the samples. To investigate the error caused by the approximate analytical solution, the air permeabilities are also estimated based on fully numerical solutions. The permeability values obtained from analytical and numerical solutions are very close. The maximum relative error is less than $6 \%$ for samples with more than five pressure-time records. A quantitative condition is given under which the analytical solution applies with negligible estimation error. Compared with the common, steady-state method for measuring air permeability, the falling-pressure method has its advantages such as simplicity and economy. The steady-state method has to measure the air flux through the sample, while the falling-pressure method does not.
\end{abstract}

(C) 2003 Elsevier B.V. All rights reserved.

Keywords: Air permeability; Air flow; Falling-pressure method; Analytical solution; Numerical solution; Inverse problem

\footnotetext{
* Corresponding author. Address: Department of Mathematics, Anshan Normal University, Anshan 114005, China. Tel.: +86-4122960398; fax: + 86-412-2960111.

E-mail addresses: hailong@graduate.hku.hk (H. Li), jjiao@ hku.hk (J.J. Jiao), lukm@ hkairport.com (M. Luk).
}

\section{Introduction}

Air permeability is one of the most important parameters in all gas flow simulations (e.g. Shan et al., 1992; Shan, 1995). The common method for determining the air permeability of unsaturated rock, soil or other materials is to measure the steady-state air flux through the soil sample (e.g. Stonestrom and 
Rubin, 1989; Springer et al., 1998). Although the data analysis of this method is simple, the experiment apparatus is complex and expensive in order to maintain and measure the steady-state air flux. Massmann and Johnson (2001) proposed a very simple method to estimate air permeability in laboratory. The main drawback of their method is that the air is treated as an incompressible fluid. This assumption can only be used when the variation range of the air pressure in the experiment is sufficiently small. This paper introduces a falling-pressure method to measure air permeability in laboratory. The air is treated as compressible fluid. An analytical solution describing the air pressure versus time relationship is derived. Test data of 15 samples are analyzed both analytically and numerically to examine the applicability of the falling-pressure method.

\section{Description of the test}

The apparatus comprises a $20 \mathrm{~cm}$ diameter cylinder in which the column-shaped sample is fixed and sealed around the perimeter with a silicone sealant. The lower surface of the sample and the cylinder form an air chamber. The upper surface of the sample is open to the atmosphere. A simple foot pump is used to pressurize the air chamber through an air inlet. Then the valve of the air inlet is closed so that the air in the air chamber can only leak out through the porous sample. A water manometer is connected to the air chamber to measure the air pressure inside the chamber (see Fig. 1). The test procedure comprises the following steps.

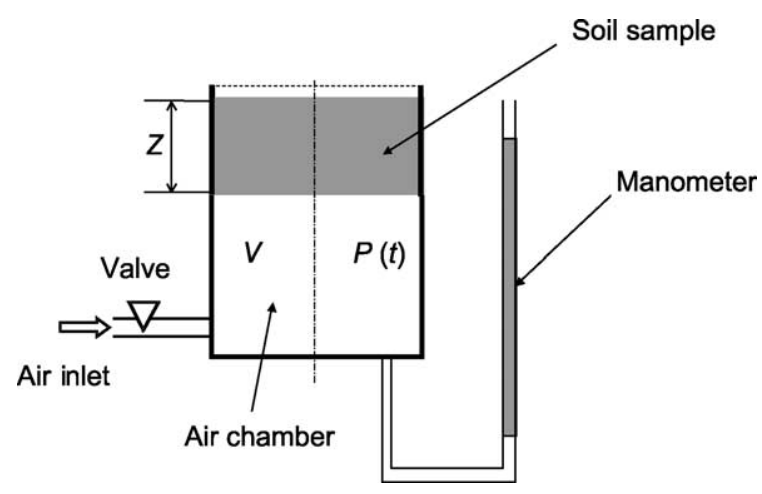

Fig. 1. Schematic of a falling-pressure air permeameter.
1. Seal a sample in the cylinder and allow $24 \mathrm{~h}$ for silicone sealant to set.

2. Increase the pressure below the sample using a foot pump until the manometer indicates a pressure slightly greater than $3 \mathrm{kPa}$.

3. Shut off the valve to foot pump. The air trapped in the air chamber can only escape by flowing through the sample.

4. The data record starts (i.e. $t=0$ ) when the manometer indicates $3 \mathrm{kPa}$. The time when the pressure in the air chamber drops by every $0.2 \mathrm{kPa}$ is recorded. The maximum number of record times when the pressure drops from 3 to $0.2 \mathrm{kPa}$ is 14 . For some samples with relatively higher air permeability the monometer reading drops so quickly that the first several monometer readings are missed. In this case, the times corresponding to the pressure values of $2.8,2.6 \mathrm{kPa}$, etc. are not available.

The equipment was designed and the test was carried out by a consulting firm. Further details on the apparatus and the test procedures can be found in GCG LTD (2001).

\section{Theoretical analysis of the pressure versus time relationship}

\subsection{Mathematical model}

If the gravitational component of the driving force is negligible in comparison to the force of the applied pressure gradient, the one-dimensional isothermal air flow through the sample can be ideally described by the extended Darcy's law for gases (Muskat, 1946, pp. 76-78; Stonestrom and Rubin, 1989)

$q(z, t)=-\frac{k}{\mu} \frac{\partial P_{\mathrm{Smpl}}}{\partial z}$.

Here $q$ is the volumetric flux $\left[\mathrm{L} \mathrm{T}^{-1}\right], k$ is the slipenhanced air permeability $\left[\mathrm{L}^{2}\right], \mu$ is the dynamic viscosity of the gas $\left[\mathrm{M} \mathrm{L}^{-1} \mathrm{~T}^{-1}\right], P_{\mathrm{Smpl}}(z, t)$ is the air-phase pressure $\left[\mathrm{M} \mathrm{L}^{-1} \mathrm{~T}^{-2}\right]$ in the sample, $z$ is the distance $[\mathrm{L}]$ along the one-dimensional air flow in the sample $(z=0$ at the interface of the sample and the sample-confined air chamber, $z=Z$ at the upper surface of the sample, see Fig. 1), $t$ is time [T]. 
According to the ideal gas law (Baehr and Hult, 1991)

$\rho=\frac{\omega P_{\mathrm{Smpl}}}{R T}$,

where $\rho(z, t)$ is the density of the air-phase [ $\left.\mathrm{M} \mathrm{L}^{-3}\right], \omega$ is the average molecular weight $\left[\mathrm{M} \mathrm{mol}^{-1}\right]$ of the airphase, $T$ is temperature [Kelvin], $R$ is the universal gas constant $\left[\mathrm{M} \mathrm{L}^{-2} \mathrm{~T}^{-2} \mathrm{~mol}^{-1} \mathrm{Kelvin}^{-1}\right]$. The mass of air-phase leaking through the sample in the time interval $[t, t+\mathrm{d} t]$ is

$\mathrm{d} m_{\text {leaky }}=A \rho(0, t) q(0, t) \mathrm{d} t$,

where $A$ is the cross-section area $\left[\mathrm{L}^{2}\right]$ of the sample in the air chamber. The mass loss of air in the sampleconfined air chamber in the time interval $[t, t+\mathrm{d} t]$ is

$\mathrm{d} m_{\mathrm{loss}}=-V \frac{\mathrm{d} \rho(0, t)}{\mathrm{d} t} \mathrm{~d} t=-V \mathrm{~d} \rho(0, t)$,

where $V$ is the volume $\left[\mathrm{L}^{3}\right]$ of the air chamber. According to the mass conservation law, $\mathrm{d} m_{\text {leaky }}=$ $\mathrm{d} m_{\text {loss }}$, so one has

$A \rho(0, t) q(0, t) \mathrm{d} t=-V \mathrm{~d} \rho(0, t)$.

Regarding temperature $T$ in Eq. (2) as constant (isothermal state) and substituting Eqs. (1) and (2) into Eq. (5), yield

$\left.\frac{A k}{2 V \mu} \frac{\partial P_{\mathrm{Smpl}}^{2}}{\partial z}\right|_{z=0}=\frac{\mathrm{d} P}{\mathrm{~d} t}$,

where $P(t)=P_{\text {Smpl }}(0, t)$ is the air pressure in the air chamber at time $t$. Eq. (6) describes the air pressure change in the air chamber.

In Eq. (24) of Baehr and Hult (1991), neglecting the gravitational effect and vertical variation of temperature, one can obtain the following equation governing the isothermal, one-dimensional, vertical and compressible air flow in the sample

$\frac{P_{\mathrm{m}}}{P_{\mathrm{Smpl}}} \frac{n_{\mathrm{a}}}{P_{\mathrm{m}}} \frac{\partial P_{\mathrm{Smpl}}^{2}}{\partial t}=\frac{k}{\mu} \frac{\partial^{2} P_{\mathrm{Smpl}}^{2}}{\partial z^{2}}$,

where $n_{\mathrm{a}}$ is the air-filled porosity (dimensionless), and $P_{\mathrm{m}}$ is the mean air pressure defined as the average of the maximum and minimum pressures in the test. As described in Section 2, the pressure $P_{\text {Smpl }}$ ranges from the minimum pressure (atmospheric pressure) $P_{\mathrm{atm}}=101.3 \mathrm{kPa}$ to the maximum pressure $P_{\text {atm }}+3.0=104.3 \mathrm{kPa}$. This gives a value of $P_{\mathrm{m}}=102.8 \mathrm{kPa}$. So the term $P_{\mathrm{m}} / P_{\mathrm{Smpl}}$ in Eq. (7a) can be approximated by 1.0 with an error less than $1.5 \%$. Therefore, Eq. (7a) can be approximately linearized as (e.g. Shan, 1995)

$\frac{n_{\mathrm{a}}}{P_{\mathrm{m}}} \frac{\partial P_{\mathrm{Smpl}}^{2}}{\partial t}=\frac{k}{\mu} \frac{\partial^{2} P_{\mathrm{Smpl}}^{2}}{\partial z^{2}}, \quad 0<z<Z$.

On the upper and lower surfaces of the sample, the air pressure $P_{\text {Smpl }}(z, t)$ satisfies the following boundary conditions

$\left.P_{\text {Smpl }}^{2}(z, t)\right|_{z=0}=P^{2}(t)$,

$\left.P_{\text {Smpl }}^{2}(z, t)\right|_{z=Z}=P_{\text {atm }}^{2}$.

When $t=0$, the air pressure $P_{\mathrm{Smpl}}(z, t)$ satisfies the initial condition

$\left.P_{\text {Smpl }}^{2}(z, t)\right|_{t=0}=P_{\text {Smpl }}^{2}(z, 0)$.

\subsection{Model simplification and analytical solution}

It is difficult to obtain the exact analytical solution of $P(t)$ from models (6), (7b) and (8)-(10). Under certain reasonable assumption, models $(6),(7 \mathrm{~b})$ and (8)-(10) can be significantly simplified. Integrating Eq. (7b) in the interval $(0, \xi)$ with respect to $z$, then integrating it again in the interval $(0, Z)$ with respect to $\xi$, and using the boundary conditions (8) and (9), yield

$$
\begin{aligned}
& \left.\frac{\partial P_{\text {Smpl }}^{2}}{\partial z}\right|_{z=0}=\frac{P_{\mathrm{atm}}^{2}-P^{2}(t)}{Z}(1-\varepsilon) \\
& \varepsilon=\frac{1}{P_{\mathrm{atm}}^{2}-P^{2}(t)} \frac{n_{\mathrm{a}} \mu}{k P_{\mathrm{m}}} \int_{0}^{Z}\left[\int_{0}^{\xi} \frac{\partial P_{\mathrm{Smpl}}^{2}}{\partial t}(z, t) \mathrm{d} z\right] \mathrm{d} \xi
\end{aligned}
$$

Differentiate both sides of Eq. (7b) with respect to time $t$, and denote $\partial P_{\text {Smpl }}^{2} / \partial t$ by $\varphi(z, t)$, it follows that

$\frac{n_{\mathrm{a}}}{P_{\mathrm{m}}} \frac{\partial \varphi}{\partial t}=\frac{k}{\mu} \frac{\partial^{2} \varphi}{\partial z^{2}}, \quad 0<z<Z$. 
Using boundary conditions (8) and (9), the boundary conditions for $\varphi$ are

$\left.\varphi\right|_{z=0}=\frac{\mathrm{d} P^{2}}{\mathrm{~d} t},\left.\quad \varphi\right|_{z=Z}=0$.

At the very initial time $t_{0}$ when one begins to pump air into the chamber, the air pressure in the chamber and sample is atmospheric, so

$\left.\varphi(z, t)\right|_{t=t_{0}}=0$.

Based on the extremum principle of the initialboundary problem of Eqs. (11c)-(11e), it follows that

$|\varphi(z, t)|=\left|\frac{\partial P_{\text {Smpl }}^{2}}{\partial t}\right| \leq\left|\frac{\mathrm{d} P^{2}}{\mathrm{~d} t}\right|=2\left|P \frac{\mathrm{d} P}{\mathrm{~d} t}\right|$,

$(0<z<Z)$.

Substitute Eq. (11f) into Eq. (11b), one obtains

$|\varepsilon| \leq \frac{n_{\mathrm{a}} \mu Z^{2}}{k P_{\mathrm{m}}} \frac{P(t)}{P^{2}(t)-P_{\mathrm{atm}}^{2}}\left|\frac{\mathrm{d} P}{\mathrm{~d} t}\right| \stackrel{\text { def }}{=} \delta$.

If $\delta \ll 1$, the term $\varepsilon$ in Eq. (11a) can be neglected and the approximate equation

$\left.\frac{\partial P_{\text {Smpl }}^{2}}{\partial z}\right|_{z=0} \approx \frac{P_{\mathrm{atm}}^{2}-P^{2}(t)}{Z}$

applies. Substituting Eq. (13) into Eq. (6), one has

$\frac{A k}{V Z \mu} \mathrm{d} t=\frac{2 \mathrm{~d} P}{\left(P_{\mathrm{atm}}+P\right)\left(P_{\mathrm{atm}}-P\right)}$.

Integrating Eq. (14) from 0 to $t$, yields

$\ln \left(c \frac{P(t)-P_{\mathrm{atm}}}{P(t)+P_{\mathrm{atm}}}\right)=-\frac{A P_{\mathrm{atm}} k}{V Z \mu} t$

where $c$ is a constant given by

$c=\frac{P(0)+P_{\mathrm{atm}}}{P(0)-P_{\mathrm{atm}}}$.

Eq. (15a) implies that $\ln \left[c\left(P-P_{\mathrm{atm}}\right) /\left(P+P_{\mathrm{atm}}\right)\right]$ is linearly dependent on time $t$. Let $s$ be the slope of the $\ln \left[c\left(P-P_{\mathrm{atm}}\right) /\left(P+P_{\mathrm{atm}}\right)\right]$ versus $t$ straight line, then from Eq. (15a), the air permeability is given by

$k=-\frac{V Z \mu s}{A P_{\mathrm{atm}}}$.

\section{Application examples}

In this section, the method introduced in Section 3.2 will be used to analyze the test data of 15 asphalt samples. These samples were collected from two different locations C402 and C409 at the runway of the Hong Kong International Airport (GCG LTD, 2001). The diameter of each samples is $18.8 \mathrm{~cm}$, so the cross-section area $A=9.4^{2} \pi=277.6 \mathrm{~cm}^{2}$. The thickness of each sample is $Z=5.5 \mathrm{~cm}$. The atmosphere pressure is taken to be $P_{\text {atm }}=101.3 \mathrm{kPa}$, and the initial pressure in the air chamber is $P(0)=$ $P_{\text {atm }}+3=104.3 \mathrm{kPa}$. The dynamic viscosity of the air is taken to be $\mu=1.76 \times 10^{-4} \mathrm{~g} \mathrm{~cm}^{-1} \mathrm{~s}^{-1}$ (Baehr and Hult, 1991). The volume $V$ of the air chamber is $1644 \mathrm{~cm}^{3}$. The test was repeated for 3 or 4 times for the each sample.

Fig. 2 shows how the pressure difference $P(t)-P_{\text {atm }}$ changes with the time for the four repeated tests done for sample $\mathrm{CH} 10$. One can see that the repeatability of the tests is very good. Fig. 3 shows how the pressure function $\ln \left[c\left(P-P_{\text {atm }}\right) /(P+\right.$ $\left.P_{\text {atm }}\right)$ ] changes with the time for the four repeated tests. For each repeated test, the recorded pressures in the air chamber are the same and their values are among $3.0,2.8, \ldots, 0.2$ and $0 \mathrm{kPa}$. So there are four groups of time records corresponding to the four repeated tests. The averages of the four groups of time records are used to determine the $\ln \left[c\left(P-P_{\text {atm }}\right) /(P+\right.$ $\left.\left.P_{\text {atm }}\right)\right]$ versus $t$ straight line by means of the linear least-squares fitting, which is shown in Fig. 3. One can see that fitting between the transformed observation data and the theoretical prediction is satisfactory. When the pressure $P(t)$ in the chamber is close to the atmosphere pressure $P_{\text {atm }}$, very small observation error of $P(t)$ can result in great error of the function $\ln \left[c\left(P-P_{\text {atm }}\right) /\left(P+P_{\text {atm }}\right)\right]$ because

$\lim _{P \rightarrow P_{\mathrm{atm}}} \ln \left[c\left(P-P_{\mathrm{atm}}\right) /\left(P+P_{\mathrm{atm}}\right)\right]=\infty$.

The observation data when $P(t)-P_{\mathrm{atm}} \leq 0.2 \mathrm{kPa}$ are therefore not used in the data analysis. Using the slope of the straight line, the air permeability of the sample 


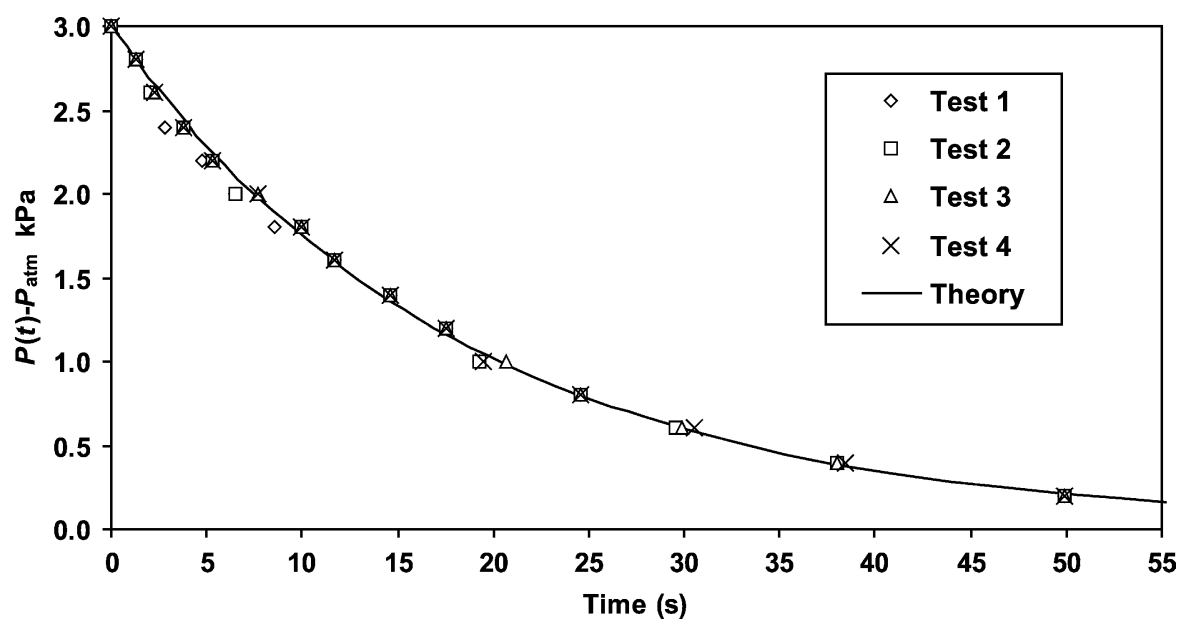

Fig. 2. $P(t)-P_{\mathrm{atm}}$ versus time test data of the sample $\mathrm{CH} 10$ and their analytical prediction by Eq. (18).

is estimated based on Eq. (16). Finally, using the estimated air permeability, the pressure versus time relation can be derived from Eqs. (15) and (16) as follows

$P(t)=\frac{\left(c+\mathrm{e}^{-s t}\right) P_{\mathrm{atm}}}{c-\mathrm{e}^{-s t}}$.

Eq. (18) gives the theoretical prediction to the pressure $P(t)$ at any time $t>0$. The solid-line in Fig. 2 shows the curve of Eq. (18) for the estimated air permeability $k$ of the sample $\mathrm{CH} 10$. One can see that the fitting between the observation data and the theoretical prediction is satisfactory.
The same analysis procedure is repeated for test data of the other samples and the results are summarized in Table 1. The column beginning with the symbol $k_{\mathrm{a}}$ in Table 1 shows the air permeability $\left(k_{\mathrm{a}}\right)$ of the 15 samples estimated by the approximate analytical solution (15a). Among the samples, sample $\mathrm{CH} 4$ has the minimum permeability of $1.18 \times 10^{-16} \mathrm{~m}^{2}$, and sample $\mathrm{CH} 9$ has the maximum permeability of $9.22 \times 10^{-13} \mathrm{~m}^{2}$. Fig. 4 shows the pressure-time test data for sample $\mathrm{CH} 4$ and their theoretical prediction based on Eq. (18). Fig. 5 shows the $\ln \left[c\left(P-P_{\mathrm{atm}}\right) /\left(P+P_{\mathrm{atm}}\right)\right]$ versus time data for the three repeated tests of the sample

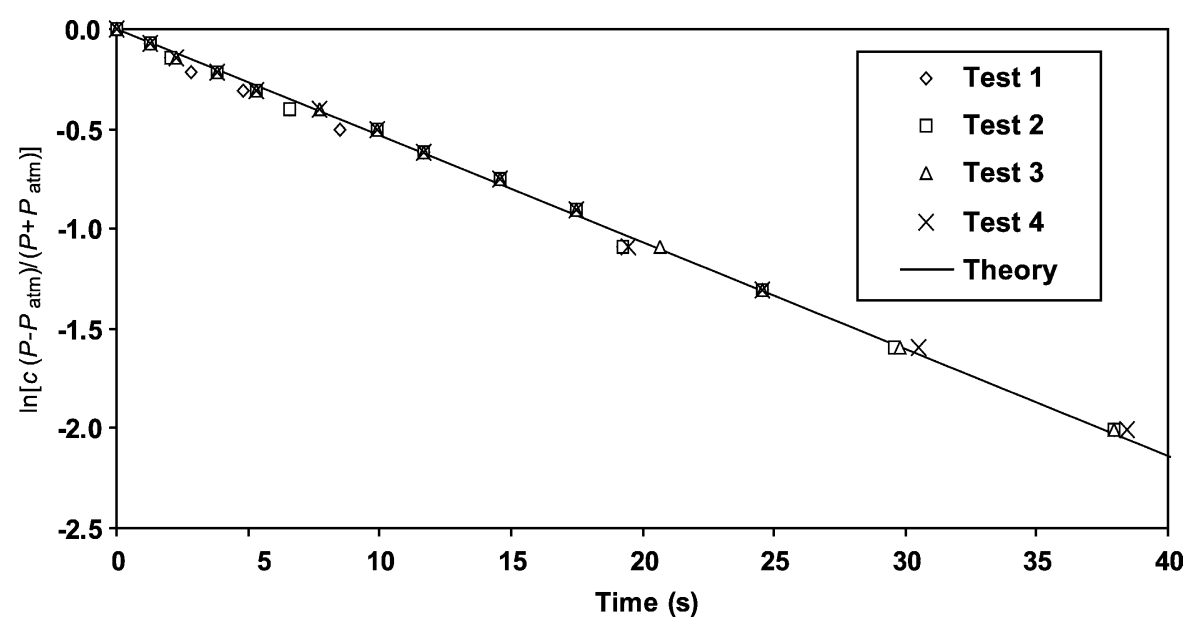

Fig. 3. $\ln \left[c\left(P-P_{\text {atm }}\right) /\left(P+P_{\text {atm }}\right)\right]$ versus time test data of the sample $\mathrm{CH} 10$ and their linear analytical prediction by Eq. (15a). 
Table 1

Air permeabilities and least-squares residuals for the 15 asphalt samples estimated by the analytical solution (15a) and numerical method when the air-filled porosity $n_{\mathrm{a}}=0.1,0.01$

\begin{tabular}{|c|c|c|c|c|c|c|c|c|c|}
\hline \multirow[t]{2}{*}{ Sample name } & \multicolumn{3}{|c|}{ Air permeability $k\left(\mathrm{~m}^{2}\right)$} & \multirow{2}{*}{$\begin{array}{l}\text { Relative } \\
\text { error }(\%)\end{array}$} & \multirow[t]{2}{*}{$\delta$} & \multicolumn{3}{|c|}{ Least-squares residual $\left(\mathrm{s}^{2}\right)$} & \multirow[t]{2}{*}{$N_{\mathrm{m}}$} \\
\hline & $\left.k\right|_{n_{\mathrm{a}}=0.1}$ & $\left.k\right|_{n_{\mathrm{a}}=0.01}$ & $k_{\mathrm{a}}$ & & & $n_{\mathrm{a}}=0.1$ & $n_{\mathrm{a}}=0.01$ & $\begin{array}{l}\text { Analytical } \\
\text { solution }\end{array}$ & \\
\hline C143 & $1.15 \times 10^{-13}$ & $1.12 \times 10^{-13}$ & $1.10 \times 10^{-13}$ & 4.51 & $7.81 \times 10^{-2}$ & 1.64 & 1.65 & 1.64 & 10 \\
\hline C144 & $4.35 \times 10^{-14}$ & $4.21 \times 10^{-14}$ & $4.21 \times 10^{-14}$ & 3.13 & $6.92 \times 10^{-2}$ & 2.94 & 3.08 & 2.99 & 14 \\
\hline $\mathrm{C} 146$ & $8.64 \times 10^{-14}$ & $8.43 \times 10^{-14}$ & $8.33 \times 10^{-14}$ & 3.61 & $6.05 \times 10^{-2}$ & 1.87 & 1.90 & 1.87 & 12 \\
\hline $\mathrm{C} 148$ & $2.16 \times 10^{-13}$ & $2.10 \times 10^{-13}$ & $2.03 \times 10^{-13}$ & 5.93 & $8.09 \times 10^{-2}$ & 2.21 & 2.24 & 2.27 & 6 \\
\hline C149 & $6.58 \times 10^{-13}$ & $6.40 \times 10^{-13}$ & $5.88 \times 10^{-13}$ & 10.64 & $7.54 \times 10^{-2}$ & 0.335 & 0.334 & 0.344 & 3 \\
\hline $\mathrm{C} 150$ & $1.59 \times 10^{-13}$ & $1.55 \times 10^{-13}$ & $1.52 \times 10^{-13}$ & 4.46 & $6.53 \times 10^{-2}$ & 0.912 & 0.912 & 0.919 & 4 \\
\hline $\mathrm{C} 151$ & $4.70 \times 10^{-14}$ & $4.61 \times 10^{-14}$ & $4.56 \times 10^{-14}$ & 2.90 & $7.02 \times 10^{-2}$ & 1.35 & 1.22 & 1.29 & 9 \\
\hline $\mathrm{C} 153$ & $3.79 \times 10^{-13}$ & $3.70 \times 10^{-13}$ & $3.47 \times 10^{-13}$ & 8.54 & $6.77 \times 10^{-2}$ & 0.397 & 0.396 & 0.407 & 3 \\
\hline $\mathrm{CH} 2$ & $1.39 \times 10^{-13}$ & $1.35 \times 10^{-13}$ & $1.33 \times 10^{-13}$ & 4.63 & $1.04 \times 10^{-1}$ & 2.80 & 2.84 & 2.83 & 9 \\
\hline $\mathrm{CH} 3$ & $8.19 \times 10^{-13}$ & $7.91 \times 10^{-13}$ & $7.42 \times 10^{-13}$ & 9.43 & $2.99 \times 10^{-2}$ & 0.00001 & 0.00003 & 0 & 1 \\
\hline $\mathrm{CH} 4$ & $1.18 \times 10^{-16}$ & $1.18 \times 10^{-16}$ & $1.18 \times 10^{-16}$ & 0.00 & $5.51 \times 10^{-2}$ & 159,000 & 159,000 & 144,000 & 13 \\
\hline $\mathrm{CH} 5$ & $4.39 \times 10^{-13}$ & $4.28 \times 10^{-13}$ & $4.00 \times 10^{-13}$ & 8.88 & $9.61 \times 10^{-2}$ & 0.998 & 1.00 & 1.03 & 4 \\
\hline CH6 & $8.35 \times 10^{-13}$ & $8.17 \times 10^{-13}$ & $7.62 \times 10^{-13}$ & 8.75 & $3.20 \times 10^{-2}$ & 0 & 0 & 0 & 1 \\
\hline $\mathrm{CH} 9$ & $1.08 \times 10^{-12}$ & $1.05 \times 10^{-12}$ & $9.22 \times 10^{-13}$ & 14.23 & $4.98 \times 10^{-2}$ & 0.155 & 0.154 & 0.161 & 2 \\
\hline $\mathrm{CH} 10$ & $3.13 \times 10^{-14}$ & $3.04 \times 10^{-14}$ & $3.04 \times 10^{-14}$ & 2.91 & $7.26 \times 10^{-2}$ & 2.69 & 2.65 & 2.68 & 14 \\
\hline
\end{tabular}

$\mathrm{CH} 4$ and the analytical straight line determined by the least-squares fitting using Eq. (15a).

The validity of the analytical method in Section 3.2 is based on the assumption of $\delta \ll 1$. This condition, however, cannot be pre-examined because in the definition of $\delta$ (Eq. (12)) there are two unknown parameters $k$ and $n_{\mathrm{a}}$. The value of the air-filled porosity $n_{\mathrm{a}}$ is usually less than $8 \%$ (Asphalt Institute, 1989, p. 285). Using an upper bound value of $n_{\mathrm{a}}=0.1$ and the values of the air permeability $k$ estimated by the approximate analytical method, a posterior estimation of the upper bound of $\delta$ can be obtained from Eq. (12). The value of $\mathrm{d} P / \mathrm{d} t$ in Eq. (12) is approximated by the difference quotient using the recorded pressure-time test data. Table 1 lists the averaged $\delta$ value of the 3-4 tests for each sample. The value of $\delta$ ranges from 0.0299 (sample CH3) to 0.104 (sample CH2). One can see that the condition $\delta \ll 1$ is satisfied. For a given

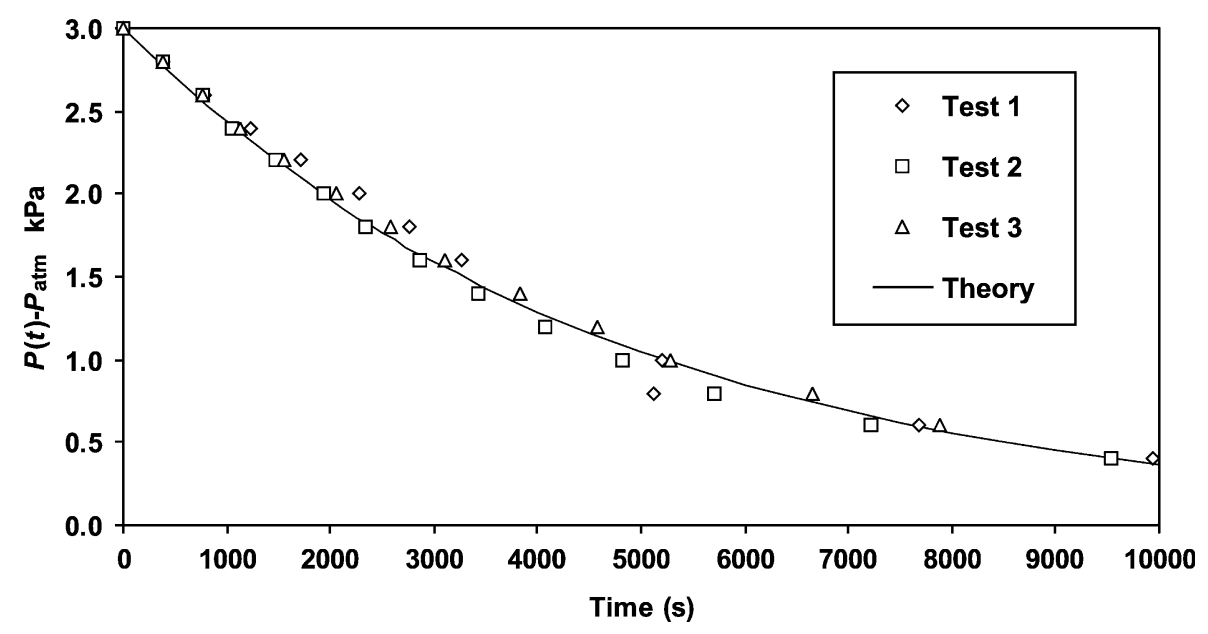

Fig. 4. $P(t)-P_{\text {atm }}$ versus time test data of the sample $\mathrm{CH} 4$ and their analytical prediction by Eq. (18). 


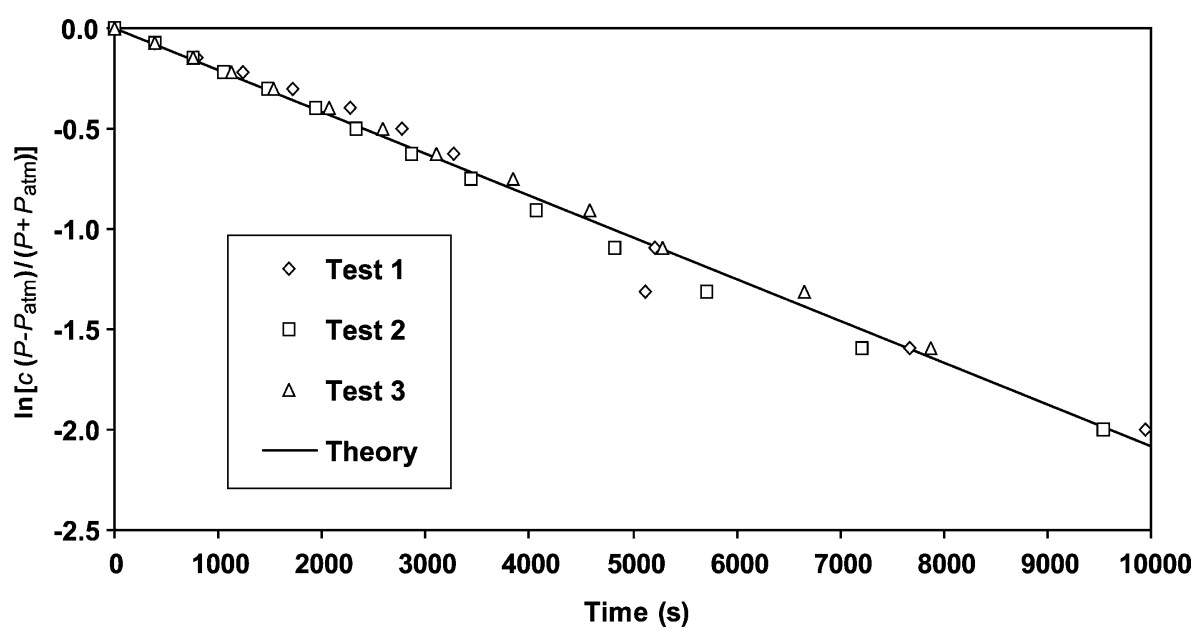

Fig. 5. $\ln \left[c\left(P-P_{\text {atm }}\right) /\left(P+P_{\text {atm }}\right)\right]$ versus time test data of the sample CH4 and their linear analytical prediction by Eq. (15a).

sample with fixed values of $k$ and $n_{\mathrm{a}}$, according to Eqs. (6) and (12), small thickness $Z$ of the sample, great volume $V$ of the air chamber or small cross-section area $A$ of the sample can result in small value of $\delta$. This conclusion is a useful instruction for the design of the test apparatus.

\section{Numerical verification}

Although the condition $\delta \ll 1$, which justifies the approximate analytical solution (15), has been verified at the end of Section 4, the verification is not straightforward and the estimation is posterior. Furthermore, the condition $\delta \ll 1$ only gives the description to the error between the exact solution of the models (6), (7b) and (8) - (10) and the approximate analytical solution (18). The quantitative error between the analytically estimated and true values of the air permeability of the sample is unknown. Due to these, a FORTRAN code is developed to estimate the air permeability of the sample based on the numerical solution of the original models $(6),(7 \mathrm{~b})$ and (8) $-(10)$ and the pressure-time test data.

In models (6), (7b) and (8)-(10), there are two unknown parameters, the air permeability $k$ and airfilled porosity $n_{\mathrm{a}}$. Given these two parameters, the pressure function $P_{\mathrm{Smpl}}(z, t)$ can be obtained by solving Eqs. (6), (7b) and (8)-(10) numerically. An iterative algorithm is performed to obtain the numerical solution of $P_{\text {Smpl }}(z, t)$ because the models
(6), (7b) and (8)-(10) is nonlinear with respect to $P_{\text {Smpl }}(z, t)$ and $P_{\text {Smpl }}^{2}(z, t)$. Initially, the pressure in the chamber equals $\left(P_{\text {atm }}+3\right) \mathrm{kPa}$. So the initial condition (10) is approximated by a linear function of the elevation, i.e.

$$
\begin{aligned}
\left.P_{\mathrm{Smpl}}^{2}(z, t)\right|_{t=0}= & P_{\mathrm{Smpl}}^{2}(z, 0) \\
= & \frac{z}{Z} P_{\mathrm{atm}}^{2}+\frac{Z-z}{Z}\left(P_{\mathrm{atm}}+3\right)^{2} \mathrm{kPa}, \\
& \quad 0 \leq z \leq Z .
\end{aligned}
$$

Let $P_{j}\left(1 \leq j \leq N_{\mathrm{m}}\right)$ be the recorded pressures in the air chamber and their values are among 2.8, $2.6, \ldots, 0.2 \mathrm{kPa} ; N_{\mathrm{m}}$ is the number of pressure data in each test for a given sample, $1<N_{\mathrm{m}} \leq 14$ (please see the fourth step of the test description). In order to estimate the air permeability of the sample based on the pressure-time data recorded in the tests, the following least-squares minimizing problem

$\min _{\log k} \sum_{j=1}^{N_{\mathrm{m}}}\left[t\left(P_{j} ; \log k, n_{\mathrm{a}}\right)-t_{j}^{*}\right]^{2}$

is solved with respect to the log-permeability $\log k$ for different fixed values of the air-filled porosity $n_{\mathrm{a}}$, where $t\left(P_{j} ; \log k, n_{\mathrm{a}}\right)$ is the time when the air pressure in the chamber equals $P_{j}$, it is determined by solving the equation $\left.P_{\mathrm{Smpl}}(z, t)\right|_{z=0}=P_{j}$ for time $t$ based on the numerical solution of $P_{\mathrm{Smpl}}(z, t), t_{j}^{*}$ is the average of the values of the time records in the three or four repeated tests for the considered sample when 
the monometer reading equals $P_{j}$. Here, the logpermeability is used instead of the permeability itself because, for nonlinear least-squares inverse problems, this log transformation can speed up the convergent speed of the iteration, avoid the computation of negative conductivities in the iteration (e.g. Carrera and Neuman, 1986).

The Gauss-Newton method is used to solve the minimizing problem (20). The sensitivity coefficient used in each Gauss-Newton iteration is approximated by parameter perturbation and numerical difference quotient method described by Yeh (1986). The main task of calculating the sensitivity coefficient is to obtain the numerical solutions of Eqs. (6), (7b) and (8)-(10) for different perturbation values of air permeability $k$.

Numerical solutions of Eqs. (6), (7b) and (8)-(10) are calculated for different initial conditions. The differences among the numerical solutions for different initial conditions decrease to zero very quickly as the time increases and become negligible when the time is equal to or greater than $t_{1} \stackrel{\text { def }}{=} t\left(P_{1}\right.$; $\left.\log k, n_{\mathrm{a}}\right)$. Therefore, the error in the approximate initial condition (19) will not influence the numerical solution of the least-squares minimizing problem (20) because each value of $t_{j} \stackrel{\text { def }}{=} t\left(P_{j} ; \log k, n_{\mathrm{a}}\right)$ included in the objective function of Eq. (20) is almost independent of the initial conditions.

Table 1 lists the numerical results of the air permeability estimations and the least-squares residuals of the minimizing problem (20) for each sample when the air-filled porosity $n_{\mathrm{a}}=0.1$ and 0.01 . The least-squares residuals for the approximate analytical solution are defined as

$\sum_{j=1}^{N_{\mathrm{m}}}\left[\frac{1}{s} \ln \left(c \frac{P_{j}-P_{\mathrm{atm}}}{P_{j}+P_{\mathrm{atm}}}\right)-t_{j}^{*}\right]^{2}$,

where $s$ is the slope of the $\ln \left[c\left(P-P_{\mathrm{atm}}\right) /\left(P+P_{\mathrm{atm}}\right)\right]$ versus $t$ straight line given by Eq. (15a), and determined by the least-squares straight-line method in Section 4. The following conclusions can be drawn from Table 1. (1) The relative error listed in Table 1, which is defined as $\left(\left.k\right|_{n_{\mathrm{a}}=0.1}-k_{\mathrm{a}}\right) /\left(\left.k\right|_{n_{\mathrm{a}}=0.1}\right)$, ranges from 0 to $5.93 \%$ for samples with more than 5 test data points $\left(N_{\mathrm{m}}>5\right)$, and from 4.46 to $14.23 \%$ for samples with less than 5 data points $\left(N_{\mathrm{m}} \leq 4\right)$. (2) For each sample, the estimated air permeability decreases with the air-filled porosity $n_{\mathrm{a}}$ as $n_{\mathrm{a}}$ ranges from 0.1 to 0.01 . Therefore, the true value of the air permeability is equal to or less than $\left.k\right|_{n_{\mathrm{a}}=0.1}$ and is equal to or greater than $\left.k\right|_{n_{\mathrm{a}}=0.01}$ because the true porosity of the sample is between 0.01 and 0.1. Due to this, the relative error $\left(\left.k\right|_{n_{\mathrm{a}}=0.1}-k_{\mathrm{a}}\right) /\left(\left.k\right|_{n_{\mathrm{a}}=0.1}\right)$ listed in Table 1 is an upper bound to the relative error between the analytically estimated air permeability $k_{\mathrm{a}}$ and the true permeability. (3) For each sample, there is no significant discrepancy among the least-squares residuals. (4) The overall consideration of above three points and the $\delta$-values in Table 1 imply that the pressure change in the air chamber will be almost independent of the air-filled porosity of the sample if the condition $\delta \leq$ 0.1 is satisfied. And consequently, if the analytical solution is used to estimate the air permeability without numerical verification, a post-examination of the assumption $\delta \leq 0.1$ can generally guarantee a relative error of less than $6 \%$ between the estimated and true values of the air permeability if the recorded pressure-time points are more than 5 . Therefore, the condition $\delta \leq 0.1$ is an applicable criterion for using the analytical solution to estimate the air permeability.

Sample CH4 has an exceptionally great residual of $1.59 \times 10^{5} \mathrm{~s}^{2}$. This great residual does not imply a bad fitting to the test data (See Fig. 4) because the time records corresponding to $P_{j}$ for sample $\mathrm{CH} 4$ are much greater than those of other samples. The residuals for other samples except $\mathrm{CH} 4$ range from approximately 0.0 (sample CH6) to $3.08 \mathrm{~s}^{2}$ (sample C144).

\section{Conclusions}

A simple analytical solution is presented to estimate air permeability using the test data obtained by a falling-pressure method in laboratory. Compared with the common method that has to measure the steady-state air flux through the soil sample, the falling-pressure method has the advantages such as simplicity and economy. It does not need to measure the air flux leaking through the sample. What is needed to record is the air pressure change with time in the air chamber. This can be easily realized and performed. The data analysis of this method is simplified significantly based on the assumption of $\delta \ll 1$ so that a straightforward method is found to estimate the air permeability using 
the pressure-time data recorded in the fallingpressure test. Physical and mathematical analyses indicate that small thickness of the sample, great volume of the air chamber or small cross-section area of the sample can result in small value of $\delta$. This conclusion is instructive for the design of the test apparatus. If $\delta \leq 0.1$, both analytical and numerical studies reveal that the pressure in the air chamber is insensitive to the air-filled porosity of the sample. As a result, the pressure function $\ln \left[c\left(P-P_{\mathrm{atm}}\right) /(P+\right.$ $\left.\left.P_{\text {atm }}\right)\right]$ decreases almost linearly with time $t$ and the air permeability of the sample is proportional to the slope of the $\ln \left[c\left(P-P_{\mathrm{atm}}\right) /\left(P+P_{\mathrm{atm}}\right)\right]$ versus $t$ straight line. By fitting the test data into a straight line in the $\ln \left[c\left(P-P_{\mathrm{atm}}\right) /\left(P+P_{\mathrm{atm}}\right)\right]$ versus $t$ plane, the air permeability can be easily estimated. The method is used to estimate the falling-pressure test data of 15 asphalt samples. The agreement between the test data and the analytical prediction is satisfactory for all the samples. The estimated values of the air permeability based on the approximate analytical solution and fully numerical solutions for different porosity values are close. Their relative error is less than $6 \%$ for samples with $N_{\mathrm{m}}>5$. All these show that the simple straight line method for estimating the air permeability in the $\ln \left[c\left(P-P_{\text {atm }}\right) /\left(P+P_{\text {atm }}\right)\right]$ versus $t$ plane is applicable and reliable if the post-estimation of $\delta$ satisfies $\delta \leq 0.1$.

\section{Acknowledgements}

This research was supported by Committee on Research and Conference Grants (CRCG) at the University of Hong Kong, the Hong Kong
Research Grants Council (RGC) of the Hong Kong Special Administration Region, China, and the National Science Foundation of China (No. 40372111). The authors are very grateful to $\mathrm{Dr}$ C. Shan and another anonymous reviewer for their valuable comments.

\section{References}

Asphalt Institute, 1989. The Asphalt Handbook, Manual Series No. 4, USA.

Baehr, A.L., Hult, M.F., 1991. Evaluation of unsaturated zone air permeability through pneumatic tests. Water Resour. Res. 27 (10), 2605-2617.

Carrera, J., Neuman, S.P., 1986. Estimation of aquifer parameters under transient and steady state conditions: 1. Maximum likelihood method incorporating prior information. Water Resour. Res. 22, 199-210.

GCG LTD, 2001. Air permeability testing. Report No. R31/6, unpublished report.

Massmann, J., Johnson, L., 2001. Exercises illustrating flow in porous media. Ground Water 39 (4), 499-503.

Muskat, M., 1946. Flow of Homogeneous Fluids through Porous Media, Second ed., J.W. Edwards, Ann Arbor, MI.

Shan, C., 1995. Analytical solutions for determining vertical air permeability in unsaturated soils. Water Resour. Res. 31 (9), 2193-2220.

Shan, C., Falta, R.W., Javandel, I., 1992. Analytical solutions for steady state gas flow to a soil vapor extraction well. Water Resour. Res. 28 (4), 1105-1120.

Springer, D.S., Loaiciga, H.A., Cullen, S.J., Everett, L.G., 1998. Air permeability of porous materials under controlled laboratory conditions. Ground Water 36 (4), 558-565.

Stonestrom, D.A., Rubin, J., 1989. Air permeability and trapped-air content in two soils. Water Resour. Res. 25 (9), 1959-1969.

Yeh, W.W-G., 1986. Review of parameter identification procedures in groundwater hydrology: the inverse problem. Water Resour. Res. 22, 95-108. 